\title{
Abordagem terapêutica nas desordens temporomandibulares: técnicas de fisioterapia associadas ao tratamento odontológico
}

Therapeutic approach in temporomandibular disorders: physical therapy techniques associated with dental treatment

Enfoque terapéutico en los desórdenes temporomandibulares:técnicas de fisioterapia asociadas a un tratamiento odontológico

Maria Cristina Rosifini ALVES-REZENDE ${ }^{\mathbf{1}}$

André Pinheiro de Magalhães BERTOZ ${ }^{1}$

Sandra Maria Herondina Coelho Ávila de AGUIAR ${ }^{1}$

Luis Guilherme Rosifini ALVES-REZENDE ${ }^{2}$

Ana Laura Rosifini ALVES-REZENDE ${ }^{3}$

Ingrid da Silva MONTANHER ${ }^{2}$

Magnum Amaral Ferreira RUIZ ${ }^{4}$ Joyce Maria VARGAS ${ }^{4}$

Rogéria Aparecida Agos FELIPE

Maria Flávia Araújo PIRES ${ }^{\mathbf{5}}$

${ }^{I}$ Departamento de Materiais Odontológicos e Prótese - Faculdade de Odontologia de Araçatuba, UNESP

${ }^{2}$ Departamento de Odontologia Infantil e Social - Faculdade de Odontologia de Araçatuba, UNESP

${ }^{3}$ Curso de Graduação em Medicina - Faculdade de Medicina da Universidade de Ribeirão Preto

${ }^{4}$ Curso de Graduação em Medicina - Faculdade de Medicina PUCCAMP

${ }^{5}$ Fisioterapeuta Voluntário - Faculdade de Odontologia de Araçatuba/Universidade Estadual Paulista "Júlio de Mesquita Filho", UNESP

${ }^{6}$ Curso de Graduação em Odontologia - Faculdade de Odontologia de Araçatuba/Universidade Estadual Paulista "Júlio de Mesquita Filho", UNESP

\section{Resumo}

Responsáveis por amplo e variado quadro de sinais e sintomas de difícil diagnóstico, as desordens da articulação temporomandibular exigem tratamento complexo e multifatorial, já que envolvem ruptura do equilíbrio biomecânico e a forte presença de componentes emocionais. Em razão de etiologia multifatorial (fatores oclusais, alterações esqueléticas, musculares, problemas degenerativos, hábitos nocivos, estresse e/ou problemas emocionais) exige abordagem terapêutica interdisciplinar por vários especialistas (cirurgião dentista, fisioterapeuta, psicólogo e fonaudiólogo)uma vez que a abordagem transdisciplinar e individualizada alicerça o tratamento bem-sucedido.Cada paciente requer procedimentos diferentes em seu tratamento, e é necessário saber em que ponto cada especialista deve intervir para o sucesso da terapêutica. As Desordens temporomandibulares (DTM), relacionadas ao desconforto na articulação temporomandibular (ATM), respondem por importante impacto negativo sobre a qualidade de vida e bem estar. São patologias multifatoriais que exigem diferentes estratégias de abordagem. Cada paciente requer procedimentos diferentes em seu tratamento, e é necessário saber em que ponto cada especialista deve intervir para o sucesso do tratamento.

Descritores: Dor Facial; Equipe de Assistência ao Paciente; Sistemas de Saúde.

\section{Abstract}

Responsible for a wide and varied picture of signs and symptoms that are difficult to diagnose, temporomandibular joint disorders require complex and multifactorial treatment, as they involve disruption of biomechanical balance and the strong presence of emotional components. Due to its multifactorial etiology (occlusal factors, skeletal, muscular changes, degenerative problems, harmful habits, stress and / or emotional problems), it requires an interdisciplinary therapeutic approach by several specialists (dental surgeon, physiotherapist, psychologist and speech therapist) as the transdisciplinary approach. and individualized underpins successful treatment. Each patient requires different procedures in their treatment, and it is necessary to know at what point each specialist should intervene for successful therapy. Temporomandibular Disorders (TMD), related to temporomandibular joint (TMJ) discomfort, account for a significant negative impact on quality of life and well-being. They are multifactorial pathologies that require different approach strategies. Each patient requires different procedures in their treatment, and it is necessary to know at what point each specialist should intervene for the success of the treatment.

Descriptors: Facial Pain; Patient Care Team; Health Systems.

\section{Resumen}

Responsables de un conjunto amplio y variado de signos y síntomas difíciles de diagnosticar, los trastornos de la articulación temporomandibular requieren un tratamiento complejo y multifactorial, ya que implican la interrupción del equilibrio biomecánico y la fuerte presencia de componentes emocionales. Debido a su etiología multifactorial (factores oclusales, esqueléticos, cambios musculares, problemas degenerativos, hábitos nocivos, estrés y / o problemas emocionales), requiere un enfoque terapéutico interdisciplinario por parte de varios especialistas (cirujano dental, fisioterapeuta, psicólogo y logopeda) como enfoque transdisciplinario. y apuntala individualmente el tratamiento exitoso. Cada paciente requiere diferentes procedimientos en su tratamiento, y es necesario saber en qué momento cada especialista debe intervenir para una terapia exitosa. Los trastornos temporomandibulares (TMD), relacionados con el malestar de la articulación temporomandibular (TMJ), representan un impacto negativo significativo en la calidad de vida y el bienestar. Son patologías multifactoriales que requieren diferentes estrategias de enfoque. Cada paciente requiere diferentes procedimientos en su tratamiento, $\mathrm{y}$ es necesario saber en qué punto debe intervenir cada especialista para el éxito del tratamiento.

Descriptores: Dolor Facial; Grupo de Atención al Paciente; Sistemas de Salud.

\section{INTRODUÇÃO}

As desordens temporomandibulares (DTMs) compreendem associação de condições médicas, dentárias e/ou faciais responsáveis por desequilíbrios na atividade do sistema estomatognático e condições dolorosas e/ou disfuncionais, que envolvem os músculos da mastigação e/ou as articulações temporomandibulares (ATM). São também denominadas distúrbios da articulação temporomandibular, perturbações da articulação temporomandibular, distúrbios craniomandibulares e desordens craniomandibulares e representam condições músculo-esqueléticas de etiologia e justificativa biológica extensas e diversificadas e, desta forma, caracterizam um grupo bastante heterogêneo de patologias ${ }^{1}$

Responsáveis por amplo e variado quadro de sinais e sintomas de difícil diagnóstico, as desordens da articulação temporomandibular exigem tratamento 
complexo e multifatorial, já que envolvem ruptura do equilíbrio biomecânico e a forte presença de componentes emocionais. São achados freqüentes dor à palpação muscular e/ou articular, função mandibular limitada e ruídos articulares, com a prevalência total em mais de $75 \%$ da população adulta e com grande impacto sobre a vida cotidiana do paciente ${ }^{1-14}$

Em razão de etiologia multifatorial (fatores oclusais, alterações esqueléticas, musculares, problemas degenerativos, hábitos nocivos, estresse e/ou problemas emocionais) exige abordagem terapêutica interdisciplinar por vários especialistas (cirurgião dentista, fisioterapeuta, psicólogo e fonaudiólogo) $)^{1,11,13}$ uma vez que a abordagem transdisciplinar e individualizada alicerça $o$ tratamento bem-sucedido ${ }^{7,15,16}$.

\section{ABORDAGEM FISIOTERÁPICA}

A fisioterapia representa um grupo de ações de suporte importante para o sucesso do tratamento de pacientes portadores de desordens temporomandibulares. A intervenção da fisioterapia é realizada através do emprego de várias modalidades terapêuticas tais como eletroterapia por ultra-som e fonoforese, laserterapia e analgesia por estimulação elétrica transcutânea (TENS), terapia de calor e frio, terapia manual para mobilização de tecidos moles e articulares bem como a liberação miofascial dependendo de uma avaliação criteriosa do paciente. De um modo geral o paciente relata alívio das condições sintomatológicas, o que permite o restabelecimento da função normal do aparelho mastigatório, além da condição postural ${ }^{1,11,13}$. Para a Academia Americana de Dor Orofacial ${ }^{2}$ a fisioterapia ajuda a aliviar a dor músculo-esquelética e a restaurar a função normal por meio da alteração da entrada sensorial, coordenando e fortalecendo a atividade muscular, e promovendo o reparo e regeneração dos tecidos.

O fisioterapeuta pode intervir não só diretamente no tratamento das DTMs, como também indiretamente na reeducação e reestruturação posturais por meio do reposicionamento da mandíbula ao crânio, minimização da dor muscular, melhora da amplitude de movimentos, reeducação do paciente em relação ao posicionamento correto da mandíbula, redução da inflamação e fortalecimento do sistema músculo-esquelético ${ }^{1,10,13,17-21}$ (Figuras 1 a 4).

Por ser a dor um dos sintomas mais comuns na DTM, pode haver variação com relação ao seu grau $^{22}$. É comum fadiga muscular e espasmos, derivados da hiperatividade muscular, que causam diminuição da coordenação motora e/ou disfunções dos músculos mastigatórios, dos músculos da cabeça e pescoço ${ }^{18,19}$.

Teixeira et al. ${ }^{23}$ preconizam a escala visual analógica (EVA) para avaliar a dor nos quadros de desordem temporomandibular. Por essa escala é possível mensurar subjetivamente desde a ausência de dor até o mais alto grau de dor ${ }^{8}$. Também são instrumentos validados para avaliação da dor o Questionário McGill de Dor (Br-MPQ) ${ }^{24}$ e o índice anamnésico (IA) proposto por Fonseca ${ }^{25}$. O primeiro, além de mensurar as diferentes qualidades da dor, fornece medidas quantitativas que podem ser tratadas estatisticamente. Além disso apresenta índices de validade e confiabilidade estabelecidos, com poder discriminativo entre os diversos componentes da dor $^{24}$. O segundo é um dos instrumentos disponíveis na língua portuguesa para a caracterização dos sintomas de DTM e foi desenvolvido para classificar os pacientes de acordo com a severidade desses sintomas $^{25}$.

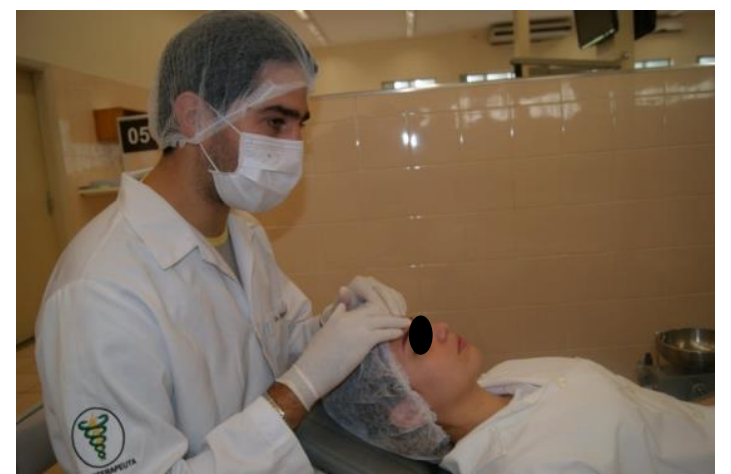

Figura 1: Terapia manual de mobilização dos músculos faciais.

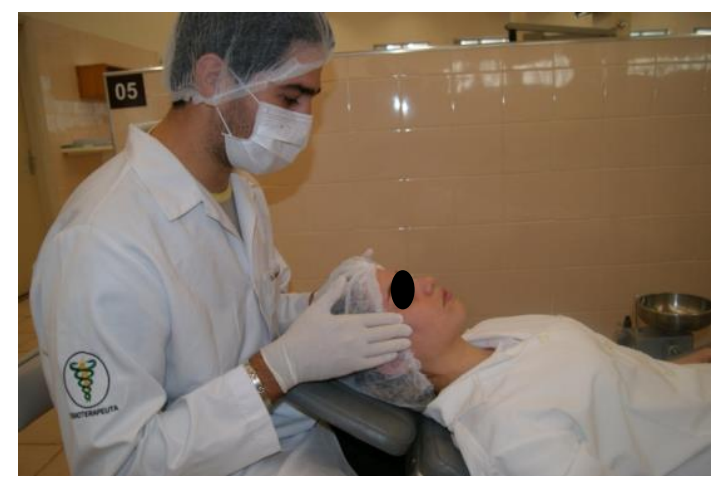

Figura 2: Terapia manual de mobilização dos músculos faciais.

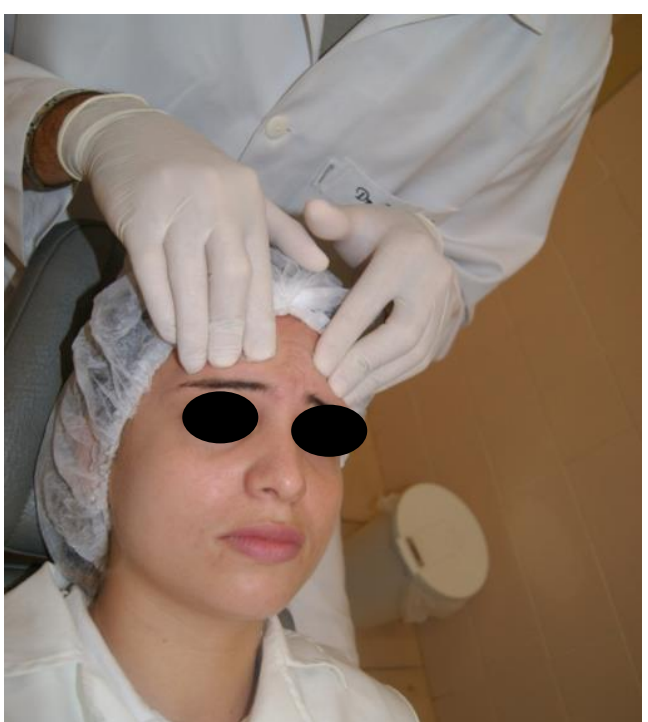

Figura 3: Terapia manual de mobilização dos músculos faciais. 


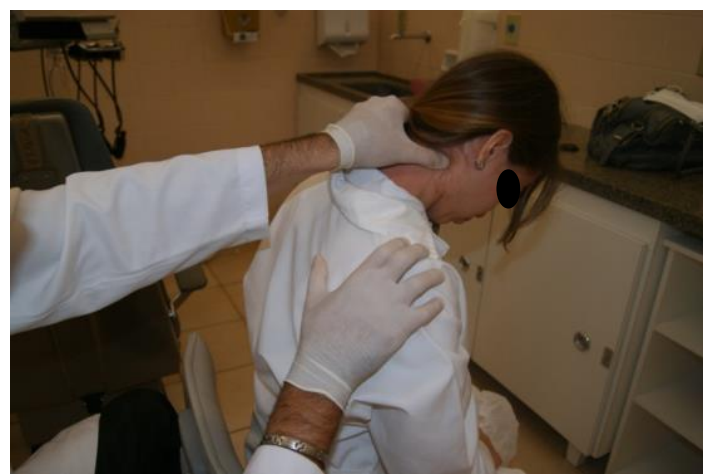

Figura 4: Terapia manual de mobilização dos músculos cervicais.

El Fatih et al. ${ }^{26}$ verificaram em seus estudos a eficácia do tratamento fisioterapêutico em 57 pacientes com desodem temporomandibular, divididos em dois grupos. Um grupo recebeu aplicações de Tens e o outro grupo recebeu aplicações de ultrassom. Ao avaliar a dor com a Escala Visual Analógica (Eva), verificou-se diminuição significativa em ambos os grupos.

Também Rodrigues et al. ${ }^{10}$ avaliaram o efeito da Tens sobre a dor de 19 indivíduos com disfunção das articulações temporomandibulares e 16 indivíduos sem sintomatologia. Observaram que os portadores de DTMs tiveram redução da dor e da atividade eletromiográfica da porção anterior do músculo temporal. Neste mesmo grupo a atividade dos músculos masseteres aumentou durante a contração máxima voluntária. Concluíram pela efetividade do Tens na redução da sintomatologia dolorosa em pacientes acometidos de desordens temporomandibulares.

Guerra $^{27}$ avaliou a eficácia do ultrassom pulsado no alívio da dor em 26 pacientes com DTM, sendo que destes, $61,53 \%$ possuíam distúrbios severos das articulações temporomandibulares e $38,46 \%$, moderados. Após o tratamento, $15.38 \%$ dos pacientes apresentavam sintomatologia severa, $34,61 \%$ moderada e $46,15 \%$ leve. $3.84 \%$ dos pacientes foram classificados como não-portadores.

Biasotto $^{28}$ estudou a eficácia da técnica de massoterapia em 60 indivíduos e verificou, por meio da Escala Visual Analógica, diminuição significativa da dor e da atividade eletromiográfica, confirmando a eficácia dessa técnica em portadores de desordem temporomandibular miogênica.

Eisensmith $^{29}$ analisou a eficácia da massagem terapêutica na redução da frequência e intensidade de sintomatologia temporomandibular. Concluiu que técnicas de massagem podem melhorar a amplitude de movimento da mandíbula, aliviar a intensidade e reduzir a frequência da dor articular. Esses achados encontram explicação em Chaitow $^{30}$ que atribui à mobilização de tecidos moles e articulares efeitos fisiológicos tais como: calmantes, estimulantes, relaxantes, neurôtonicos e circulatórios.
Troian $^{31}$ investigou a eficácia do tratamento integrado fisioterapia/odontologia ao odontológico no quadro álgico de pacientes com DTM e encontrou diminuição mais evidente da intensidade de dor nos pacientes que receberam terapia associada.

Mourão e Mesquita ${ }^{32}$ lembram que, na Odontologia, e especialmente nos pacientes com desordens temporomandibulares, o uso da fisioterapia na tem caráter reversível e visa devolver a função da articulação comprometida.

\section{CONSIDERAÇÕES FINAIS}

A fisioterapia tornou-se parte integral da abordagem interdisciplinar preconizada no tratamento da dor e da disfunção associadas com a desordem temporomandibular e outras condições de dor bucofacial. Representada por um grupo de ações de suporte, importante para o sucesso do tratamento do pacientes com disfunção da articulação temporomandibular. Seu leque de opções inclui massoterapia, cinesioterapia, termoterapia e eletroterapia, proporcionando, não só alívio da sintomatologia, como também restabelecimento da atividade muscular e equilíbrio postural.

\section{REFERÊNCIAS}

1. Alves-Rezende MCR, Silva JS, Soares BS, Bertoz FA, Oliveira DTN, Alves-Claro APR. Estudo da prevalência de sintomatologia temporomandibular em universitários brasileiros de Odontologia. Rev Odontol Araçatuba 2009; 30(1): 9-14.

2. Alves-Rezende MCR, Cortiglio S, Sant'anna CBM, Alves-Rezende LGR, Montanher IS, Alves-Rezende ALR. Aplicação da acupuntura no tratamento da Síndrome de Costen: relato de caso clínico. Arch Health Invest 2012; 1(Spec): 15 .

3. Alves-Rezende MCR, Sant'Anna CBM, Capalbo $\mathrm{BC}$, Zuim PRJ. Intervenção interdisciplinar no tratamento do paciente com dor orofacial: uso de acupuntura. Rev Odontol Unesp 2012; 41: 181

4. Alves-Rezende MCR, Sant'Anna CBM, Verri ACG, Cunha-Correia AS, Aguiar SMHCA, Bertoz APM, et al. Sinais e sintomas na Síndrome de Costen associada a desordens temporomandibulares: relato de caso clínico. Rev Odontol Araçatuba 2011; 32(1):65-9.

5. Alves-Rezende MCR, Soares BMS, Silva JB. Frequência de hábitos parafuncionais: estudo transversal em acadêmicos de Odontologia. Rev Odontol Araçatuba 2009; 30:59-62.

6. Cortiglio S, Alves-Rezende MCR, AlvesRezende LGR, Montanher IS, Alves-Rezende ALR. Estudo da associação entre bruxismo, consumo de álcool e tabaco em universitários brasileiros Arch Health Invest 2012; 1 (Spec): 36. 
7. Koopman JS, Huygen FJ, Dieleman JP, Mos M, Sturkenboom MC. Pharmacological treatment of neuropathic facial pain in the dutch general population. J Pain, 2009; 11(3): $264-72$.

8. Oliveira AS, Bermudez CC, Souza RA, Souza CMF, Dias EM, Castro CES, et al. Impacto da dor na vida de portadores de disfunção temporomandibular. J Appl Oral Sci 2003; 11(2): 138-43.

9. Reisine ST, Fertig J, Weber J, Leder S. Impact of dental conditions on patients' quality of life. Community Dent Oral Epidemiol 1989; 17(1): 7- 10.

10. Rodrigues D, Siriani AO, Bérzin F. Effect of conventional TENS on pain and electromyographic activity of masticatory muscles in TMD patients. Braz Oral Res 2004;18(4):290-5.

11. Rossi AC, Resende MCRA, Araújo OP Jr, Garcia AR, Zuim PRJ, Marinho LVD. Fisioterapia no tratamento multidisciplinar da disfunção temporomandibular. Rev Odonto UNESP. 2008;37(Número Especial):190

12. Seligman ME, Schulman P, Tryon AM. Group prevention of depression and anxiety symptoms. Behav Res Ther 2007; 45:1111-26.

13. Simi MD, Alves-Rezende MCR, Ruiz MAF, Zuim PRJ. Abordadem extensionista multidisciplinar: fisioterapia aplicada ao tratamento de disfunção temporomandibular. Rev Odontol UNESP. 2012;41(Número Especial): 180 .

14. Widmer CG. Convicções correntes e diretrizes pedagógicas. In: Lund JP, Lavigne GJ, Dubner R, Sessle BJ. Dor orofacial: da ciência básica à conduta clínica. São Paulo: Quintenssence Books: 2002. p. 27-34.

15. Granja DVA, Lima AP. Influência dos recursos fisioterapêuticos nas algias orofaciais. Rev Fisioter Brasil 2003;4(6):394-401.

16. Pereira Jr FJ, Vieira AR, Prado R, Miasato JM. Visão geral das desordens temporomandibulares. RGO 2004;52(2):117-21.

17. Barbosa GAS, Barbosa KVMS, Badaró CR Filho. Recursos fisioterápicos disponíveis para o tratamento das disfunções temporomandibulares. JBA 2003; 3(11):257-62.

18. Castro FM, Gomes RCV, Salomão JR, Abdon APV. A efetividade da terapia de liberação posicional (TLP) em pacientes portadores de disfunção temporomandibular. Rev Odont Univ Cidade São Paulo. 2006; 18(1):67-74.

19. Maciel RN. Oclusão e ATM: procedimentos clínicos. São Paulo: Ed. Santos; 1998. Maluf SA, Moreno BGD, Alfredo PP. Exercícios terapêuticos nas desordens temporomandibulares: uma revisão de literatura. Fisioter Pesq. 2008; 15(4): 408-15.
20. Medlicott MS, Harris SR. A systematic review of the effectiveness of exercise, manual therapy, electrotherapy, relaxation training, and biofeedback in the management of temporomandibular disorder. Physical Ther 2006; 86(7):955-73.

21. Torres F, Campos LG, Fillipini HF, Weigert KL, Dalla Vecchia GF. Efeitos dos tratamentos fisioterapêutico e odontológico em pacientes com disfunção temporomandibular Fisioter Mov 2012; 25(1):117-25

22. Teixeira MJ, Teixeira WGJ, Santos FPSS, Andrade DCA, Bezerra SL, Figueirdo JB, et al. Epidemiologia clínica da dor músculoesquelética. Rev Med 2001; 80(1): 1-21.

23. Melzack R. The McGill pain questionnaire. From description to measurement. Anesthes 2005; 103(1):199-202

24. Chaves TC, Oliveira AS, Grossi DB. Principais instrumentos para avaliação da disfunção temporomandibular, parte I: índices e questionários; uma contribuição para a prática clínica e de pesquisa. Fisioter Pesq. 2008;15(1):92-100

25. El Fatih I A, Ibrahim AI, El Laithi A. Efficacy of physiotherapy and intraoral splint in the management of temporomandibular disorders. Saud Dent J 2004;16(1):16-20.

26. Guerra LMC. Eficácia do ultra-som na terapia das Disfunções Temporomandibulares: avaliação clínica e eletromiográfica. [dissertação]. Pernambuco: Universidade Federal de Pernambuco; 2003.

27. Biasotto DA. Efeito da técnica Fisioterapeutica (massoterapia) em indivíduos portadores de Desordem Temporomandibular Miogênica: um estudo eletromiográfico. [dissertação]. Piracicaba: Faculdade de Odontologia de Piracicaba da Universidade Estadual de Campinas; 2002.

28. Eisensmith LP. Massage therapy decreases frequency and intensity of symptoms related to temporomandibular joint syndrome in one case study. J Bodywork Move Therap 2007;11:22330.

29. Chaitow L. Teoria e prática da manipulação craniana: abordagens em tecidos ossos e mole. São Paulo: Manole, 2001.

30. Troian MA. Tratamento interdisciplinar entre fisioterapia e odontologia na redução da dor em pacientes com disfunção do sistema craniocervicomandibular. Reabilit. 2005;7(26):29-39.

31. Mourão NLA, Mesquita VT. A Importância da fisioterapia no tratamento das disfunções da Atm. Terapia Manual. 2006; 4 :66-9. 


\section{CONFLITO DE INTERESSES}

Os autores declaram não haver conflitos de interesse.

\section{AUTOR PARA CORRESPONDENCIA}

\section{Maria Cristina Rosifini Alves Rezende}

rezende@foa.unesp.br

Submetido em 30/11/2012

Aceito em 15/12/2012 